NOTICE: this is the author's version of a work that was accepted for publication in Behavioural Processes.

Changes resulting from the publishing process, such as peer review, editing, corrections, structural formatting, and other quality control mechanisms may not be reflected in this document. Changes may have been made to this work since it was submitted for publication.

doi:10.1016/j.beproc.2011.10.002

\title{
Intertemporal choice in lemurs
}

\author{
Jeffrey R. Stevens ${ }^{\mathrm{a}, \mathrm{b}, *}$, Nelly Mühlhoff ${ }^{\mathrm{a}}$ \\ ${ }^{a}$ Center for Adaptive Behavior and Cognition, Max Planck Institute for Human Development, Lentzeallee 94, 14195, Berlin, Germany \\ ${ }^{b}$ Department of Psychology, University of Nebraska-Lincoln, 238 Burnett Hall, Lincoln, Nebraska 68588, USA
}

\begin{abstract}
Different species vary in their ability to wait for delayed rewards in intertemporal choice tasks. Models of rate maximization account for part of this variation, but other factors such as social structure and feeding ecology seem to underly some species differences. Though studies have evaluated intertemporal choice in several primate species, including Old World monkeys, New World monkeys, and apes, prosimians have not been tested. This study investigated intertemporal choices in three species of lemur (black-and-white ruffed lemurs, Varecia variegata, red ruffed lemurs, Varecia rubra, and black lemurs, Eulemur macaco) to assess how they compare to other primate species and whether their choices are consistent with rate maximization. We offered lemurs a choice between two food items available immediately and six food items available after a delay. We found that by adjusting the delay to the larger reward, the lemurs were indifferent between the two options at a mean delay of $17 \mathrm{~s}$, ranging from 9-25 s. These data are comparable to data collected from common marmosets (Callithrix jacchus). The lemur data were not consistent with models of rate maximization. The addition of lemurs to the list of species tested in these tasks will help uncover the role of life history and socio-ecological factors influencing intertemporal choices.
\end{abstract}

Keywords: intertemporal choice, primate, prosimian, rate maximization, temporal discounting

\section{Introduction}

The black-and-white ruffed lemur dangles by her hind legs at the top of the leafy canopy, plucking figs from the branch below. At some point she has consumed many of the figs from the branch, leaving a few small fruits hidden beneath the leaves. The lemur now faces a choice: should she continue to search for the remaining figs or move on to another branch full of fruit? These questions of when to leave a patch (Charnov, 1976; Stephens and Krebs, 1986) are an example of an intertemporal choice-a choice between options with different time delays to reward (Read, 2004; Stevens, 2010).

Intertemporal choices are ubiquitous in the lives of humans and other animals. When deciding when and where to forage, with whom to interact or mate, and

\footnotetext{
*Corresponding author's address:

Department of Psychology

University of Nebraska-Lincoln

238 Burnett Hall

Lincoln, NE 68588-0308

Phone: +1 4024726931 FAX: +1 4024724637

Email addresses: jeffrey.r.stevens@gmail.com (Jeffrey R Stevens), gefunkt@gmx.de (Nelly Mühlhoff)
}

where to establish a territory, individuals must choose between options whose benefits are available at different points in time. Historically, most experimental studies of intertemporal choices in animals tested primarily pigeons and rats (McDiarmid and Rilling, 1965; Richards et al., 1997). More recently, however, researchers have tested many more species, including insects, fish, birds, mice, and primates (Bateson and Kacelnik, 1996; Tobin et al., 1996; Brunner and Hen, 1997; Cheng et al., 2002; Stevens et al., 2005a; Rosati et al., 2007; Pearson et al., 2010; Vick et al., 2010; Mühlhoff et al., 2011).

Many studies of intertemporal choice in animals assume that they temporally discount the options (Mazur, 1987; Green and Myerson, 2004). Temporal discounting occurs when an individual assigns a subjective present value to a future reward, and that value decreases as the time delay to receiving that reward increases. In the hyperbolic discounting model (Mazur, 1987), value decreases with the reciprocal of time:

$$
V=\frac{A}{1+k d},
$$

where $A$ represents the amount of the food reward, $d$ represents the time delay to receive the reward, and $k$ represents a discounting parameter. This hyperbolic 
model describes the intertemporal choices of animals quite well (Green and Myerson, 2004). Most of the animal studies, however, offer subjects repeated instances of the same choice. An alternative to the discounting account proposes that, rather than generating a subjective value, subjects simply use reinforcement rate (Skinner, 1938) or food intake rate (Stephens and Krebs, 1986) to make these decisions (Kacelnik, 2003; Stevens and Stephens, 2009). Two different types of rates are possible, depending on which temporal components individuals use: short-term or long-term rates. Short-term rates are defined as

$$
R_{S T}=\frac{A}{d+h},
$$

where $h$ represents the handling time required to process and consume the reward. Blue jays (Cyanocitta cristata) and cotton-top tamarins (Saguinus oedipus) may use short-term rates to make intertemporal choices (Stephens and Anderson, 2001; Stevens et al., 2005a). For the long-term rate

$$
R_{L T}=\frac{A}{d+h+I T I},
$$

the time intervals after choice and consumption (here referring to the intertrial interval or ITI) are also included in the rate calculation. Though early work in pigeons suggested that the intertrial interval played little role in their choices (Mazur, 1994), rhesus macaques (Macaca mulatta) use post-choice information when it is made more salient to them (Pearson et al., 2010). Moreover, intertemporal choices in bonobos (Pan paniscus) are consistent with using long-term rates (Rosati et al., 2007). Once individuals estimate a rate for each option, they must then apply a decision rule such as matching (distributing responses to options in proportion to their relative rates-e.g., an option with twice the reward rate will receive twice as many responses, Herrnstein, 1961) or maximizing (always choosing the option that offers the highest rate, Stephens and Krebs, 1986). Combining the valuation or rate with a decision rule results in a choice.

Researchers have investigated intertemporal choice in several primate species using a variety of methodologies (Beran et al., 1999; Ramseyer et al., 2006; Pearson et al., 2010). Tobin et al. (1996), Stevens et al. (2005a), Rosati et al. (2007), and Addessi et al. (2011) have used the same adjusting-delay 'self-control' paradigm to test intertemporal choices in Old World monkeys, New World monkeys, and apes. In this task, subjects make binary choices between two immediately available food items and six food items available after a time delay. By adjusting the delay to the larger reward until subjects choose equally between the two options, researchers can establish an indifference point for each subject. To our knowledge, however, prosimian species have not been tested in an intertemporal choice task. The aim of this study was to test lemurs in this task, thereby adding the more phylogenetically basal prosimian group to the comparative data. Lemurs are good species to test because we already have data on their numerical discrimination (Lewis et al., 2005; Santos et al., 2005), inhibitory control (Genty et al., 2004), and risk sensitivity (MacLean et al., 2011). Therefore, this study will contribute to our emerging understanding of prosimian cognition and decision making. Our study examined two research questions:

1. How does lemur intertemporal choice compare to other species?

2. Do lemurs maximize their intake rate?

To explore these questions, we tested several species of lemurs in an adjusting-delay intertemporal choice procedure.

\section{Methods}

\subsection{Subjects}

In this experiment, we tested twelve lemurs of four different species at the Tierpark Berlin: four blackand-white ruffed lemurs (Varecia variegata), two red ruffed lemurs (Varecia rubra), four red-bellied lemurs (Eulemur rubriventer) and two black lemurs (Eulemur macaco). Only five lemurs (three black-and-white ruffed, one red ruffed, and one black lemur) completed the experiment. The remaining subjects failed to pass the number discrimination phase $(N=2)$, stopped responding to the choices in the titration phase $(N=4)$, or were moved into inaccessible facilities $(N=1)$. We tested the ruffed lemurs from Nov 2007 to Dec 2008 and the black lemur from May to July 2009.

None of the lemurs had prior experience with experiments. Zookeepers fed them in the early morning approximately three hours prior to our testing and in the afternoon approximately one hour after our testing. The lemurs had constant access to water.

\subsection{Materials}

For testing, we separated pair-housed individuals. The testing apparatus (Figure 1) attached to openings of the cages that were used for feeding. The apparatus consisted of a platform that was affixed horizontally to the grating outside the cage. An opaque box covered 
the top and sides of the platform. The box was open on the experimenter's side but covered by a transparent Plexiglas barrier with two holes on the subject's side. In addition, the experimenter inserted two opaque barriers into the box near the Plexiglas barrier to prevent physical and visual access to the contents of the box. Rewards were placed on two slides on either side of the box. To choose a reward, subjects reached through one of the holes. The experimenter dispensed the chosen rewards by pushing the respective slide towards the subject. We used apple pieces (about $1.5 \mathrm{~g}$ ) as rewards because they were a preferred food item and did not interfere with the subjects' diet. The experimenter used a stopwatch to measure the time intervals and videotaped all trials for behavioral analysis.

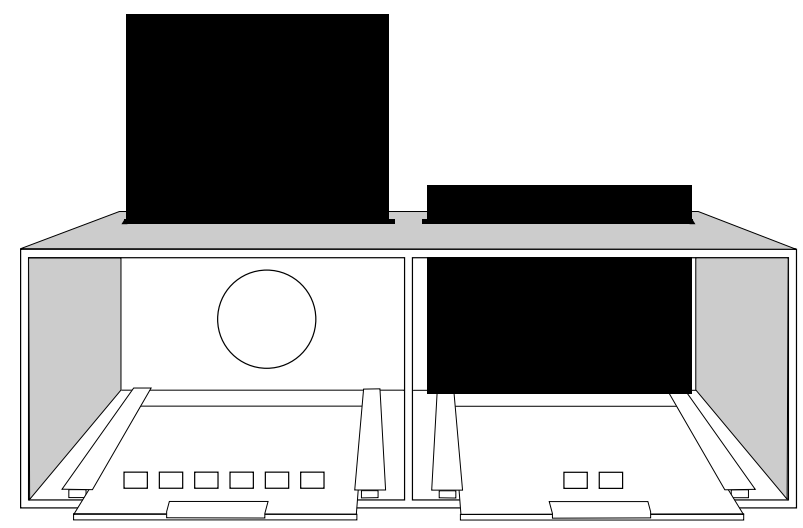

Figure 1: Experimental apparatus (experimenter view). The experimental platform attached to the subjects home cage and consisted of an opaque cover (grey), two opaque barriers (black), and two slides. The barriers prevented visual access to the rewards during the intertrial interval. Once the experimenter removed the barriers, the subject could view the options and reach through one of the two holes to make a choice. After the appropriate delay, the experimenter slid the chosen reward into the subject's reach and removed the unchosen reward.

\subsection{Experimental phases}

All tests occurred between 1100 and $1300 \mathrm{~h}$ about five days a week, with one session per subject per day. Subjects experienced three experimental phases: training, number discrimination, and titration. In each phase, subjects experienced a daily session of 14 trials. Within a session, intertrial intervals (ITIs) of 30 seconds separated trials. During the ITI, the placement of the opaque barriers prevented the subject from seeing inside the box. The reward slides remained pulled back towards the experimenter. The experimenter stood beside the cage with her back to the cage during this period. After $20 \mathrm{~s}$, the experimenter placed the rewards for the upcoming trial on the two reward slides out of sight of the subject. Reward side assignments were randomized across trials. Once the ITI elapsed, the experimenter pushed the slides forward and removed both opaque barriers simultaneously. Subjects had 15 s to examine the options and choose by reaching into one of the two holes. If they failed to choose an option within $15 \mathrm{~s}$, the experimenter recorded the trial as invalid and replaced the barriers. Once subjects made a choice, the experimenter removed the unchosen reward and pushed the chosen reward slide forward into the subject's reach after the programed delay elapsed. When the subject had removed all food pieces from the slide (food dropped on the floor was regarded as consumed), the next ITI began.

\subsubsection{Training phase}

The training phase familiarized the subjects to the general testing procedure and trained them to examine their options and make a choice. In the training phase, subjects experienced only forced-choice trials - that is, subjects chose between either zero versus two rewards or zero versus six rewards, with counterbalanced order of trial types and side assignments. In this phase, subjects received the rewards immediately after choosing an option, and thereby learned about the different reward quantities available. Subjects had to make a choice in 12 of the 14 trials for the session to be considered valid, and they had to repeat invalid sessions. To enter the next experimental phase, subjects needed to choose correctly in 12 of 14 trials for three consecutive sessions. In this phase, we scored a correct choice as reaching only into the chamber that contained the reward (either two or six food pieces).

\subsubsection{Number discrimination phase}

In the number discrimination phase, we wanted to ensure that the subjects discriminated between the two reward amounts. We offered them the choice between the smaller reward (two apple pieces) and the larger reward (six apple pieces), with side assignments counterbalanced within a session. Subjects received the rewards immediately after choosing an option. The two versus six comparison is within the lemurs' numerical discrimination abilities (Lewis et al., 2005). As in the training phase, subjects needed to choose correctly in 12 of 14 trials for three consecutive sessions to enter the next experimental phase. Subjects chose correctly when reaching for the larger reward.

\subsubsection{Titration phase}

In the titration phase, we investigated how long the lemurs would wait for the larger option. We based the 
experimental procedures on Stevens et al. (2005a). Subjects could choose between two or six apple pieces, but, in contrast to the previous phase, we introduced a delay to obtain the larger reward. That is, now the experimenter waited for a time delay to elapse before pushing the slide with the larger reward towards the subject. When entering the titration phase, this delay was zero seconds (identical to number discrimination phase), but we then stepwise increased or decreased the delay over the course of the experiment. In contrast, the smaller rewards were always available immediately. Thus, subjects chose between two rewards available immediately and six available after a delay. We counterbalanced the reward side assignments.

Sessions contained two trial types: forced- and freechoice trials. In the forced-choice trials, subjects chose between zero versus two or zero versus six apple pieces with the appropriate delay. We used this trial type to remind the subjects of the contingencies of the task. Each session contained four such trials (two of each option), with two at the beginning of each session and two randomly distributed in the following twelve trials. The remaining ten trials were free-choice trials, in which subjects could choose between the smaller and the larger reward (see Movie S1).

The experimenter adjusted the delay to the larger reward between sessions based on the subject's performance in the previous session. If the subject chose the larger reward seven to ten times in the free-choice trials, then the delay was incremented by one second. If the subject chose the larger reward four to six times, the delay remained the same. If the subject chose the larger reward zero to three times, the delay was shortened by one second. This adjustment procedure continued until the subjects became indifferent between the two options.

\subsection{Data analysis}

We considered subjects to be indifferent between the two rewards options when the mean delay of the last five sessions did not differ from the mean delay of the preceding five sessions by more than $10 \%$ (Stevens et al., 2005a; Rosati et al., 2007). Subjects stopped the experiment after achieving indifference. We used the mean delay of the last five sessions for each subject as the primary dependent variable, the mean adjusted delay to indifference.

In addition to choice behavior, we measured temporal variables of choice, including the latency to retrieve the food and the handling time. We measured retrieval latency from the time the experimenter pushed the chosen slide forward until the subject placed the first piece of food in his/her mouth. We measured handling time as the time between the subject placing the first and last piece of food into his/her mouth. We randomly selected six sessions for each subject and measured the retrieval latency and handling time in all four forced-choice trials in each session. If these data were not available because the subject did not choose on the trial or we could not assess the appropriate start and stop times from the video, we randomly selected other sessions until we had temporal data in 24 trials (12 for the smaller option and 12 for the larger option).

For a comparative analysis, we used the mean adjusted delay from 12 animal species. Seven species were tested in a manner similar to this experiment. For these species, the subjects chose between two food items available immediately and six food items after an adjusting delay. In addition to the lemurs, this included cotton-top tamarins (Saguinus oedipus) and common marmosets (Callithrix jacchus, Stevens et al., 2005a), long-tailed macaques (Macaca fascicularis, Tobin et al., 1996), brown capuchins (Cebus apella, Addessi et al., 2011), and bonobos (Pan paniscus) and chimpanzees (Pan troglodytes, Rosati et al., 2007). In addition, seven species were tested with slightly different methodologies. Amici et al. (2008) tested brown capuchins (some of the same subjects as Addessi et al., 2011), black-handed spider monkeys (Ateles geoffroyi), longtailed macaques, lowland gorillas (Gorilla gorilla), and orangutans (Pongo pygmaeus), using the same techniques as used here except the subjects chose between one and three food items. Perry et al. (2004) tested Wistar rats (Rattus norvegicus) also with one versus three food items. Finally, Green et al. (2007) tested White Carneau pigeons (Columba livia) with an adjustingdelay procedure in which the subjects chose between 5 immediate versus 30 delayed food items, and the adjustments occurred within a session. We estimated the pigeons' mean adjusted delay to the larger reward by substituting each subject's discounting parameter $k$ into a set of hyperbolic equations (equation 1) and solving for the delay to larger reward. Body mass for each species was taken from Kangas and Branch (2006) for pigeons, Perry et al. (2004) for rats, and Isler et al. (2008) for all other primate species.

We analyzed the data using $\mathrm{R}$ statistical software version 2.13.2 (R Development Core Team, 2011) and the ape version 2.7-3 (Paradis et al., 2004), beeswarm version 0.1.1 (Eklund, 2010), and xtable version 1.5-6 (Dahl, 2009) packages. Data and R code are available in the Supplementary Materials (Data S1-S3), and data are available at the Dryad data repository (doi:10.5061/ dryad.fb778jf2). The original $\mathrm{LT}_{\mathrm{E}} \mathrm{X}$ document, with Sweave-embedded R code (Leisch, 2002) to allow re- 
production of analyses (de Leeuw, 2001), is available from JRS.

\section{Results}

Subjects required between 6-38 sessions (mean = 14.8 , median $=9$ ) to pass the number discrimination phase. In the titration phase, the mean adjusted delay at indifference observed for the lemurs tested in this study ranged from 9-25 s, with a mean of $17 \mathrm{~s}$. Table 1 shows the mean adjusted delay for the individual subjects. There do not appear to be strong differences between lemur species (the minimum and maximum delays both come from the black-and-white ruffed lemurs), but we tested too few subjects to make proper between-species comparisons. Thus, we pool all of the lemur species in the subsequent comparative analyses.

Previous studies with cotton-top tamarins and bonobos suggest that their intertemporal choice patterns are consistent with short-term and long-term rate maximization, respectively (Stevens et al., 2005a; Rosati et al., 2007). To investigate whether lemur intertemporal choice is consistent with maximizing intake rate, we measured individual response latencies and handling times for both the smaller and larger rewards (Table 1). The inclusion or exclusion of retrieval latency did not influence the results, so all analyses added retrieval latency to the handling time. If lemurs maximize their short-term intake rate, they should choose the option with the higher rate (Equation 2). We can solve the equation $\frac{A_{s}}{d_{s}+h_{s}}=\frac{A_{l}}{d_{l}+h_{l}}$ (where the subscripts $s$ and $l$ refer to the smaller and larger option, respectively) for $d_{l}$ to generate a predicted mean adjusted delay at indifference for each subject. The mean short-term rate maximization prediction for the adjusted is $-4.9 \mathrm{~s}$ (range -13.09 to $0.24 \mathrm{~s}$ ), clearly not matching the observed mean of $17.0 \mathrm{~s}$. The long-term rate (Equation 3) includes the intertrial interval (ITI) of $30 \mathrm{~s}$. When solving $\frac{A_{s}}{d_{s}+h_{s}+I T I}=\frac{A_{l}}{d_{l}+h_{l}+I T I}$, the mean long-term rate maximization prediction for the adjusted delay is $55.1 \mathrm{~s}$ (range 46.91 to $60.24 \mathrm{~s}$ ), again not matching the observed adjusted delay.

\section{Discussion}

Using an adjusting-delay procedure in three species of lemur, we measured the mean adjusted delay to indifference such that subjects were indifferent between two food items received immediately and six food items received after a delay. The observed mean adjusted delays ranged from 9-25 s. Relative to other species tested
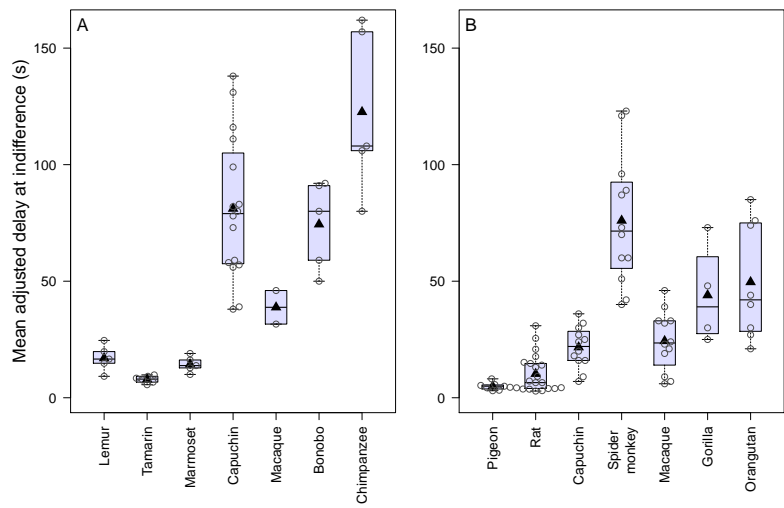

Figure 2: Intertemporal choice across animal species. (A) Seven species have been tested using the same adjusting-delay procedure with two versus six food items: lemurs (this study), cotton-top tamarins and common marmosets (Stevens et al., 2005a), brown capuchins (Addessi et al., 2011), long-tailed macaques (Tobin et al., 1996), and bonobos and chimpanzees (Rosati et al., 2007). The mean adjusted delay at indifference for lemurs matched that found in common marmosets. (B) Other studies used similar but not identical adjusting-delay procedures for seven species: White Carneau pigeons (Green et al., 2007), Wistar rats (Perry et al., 2004), and brown capuchins, black-handed spider monkeys, long-tailed macaques, lowland gorillas, and orangutans (Amici et al., 2008). The capuchins and macaques are the same species in both panels, and some of the capuchin data are from the same individuals in both panels. In this figure, circles represent data points for individual subjects, triangles represent the mean, lines represent the median, boxes represent the interquartile range $(25-75 \%)$, and whiskers represent the range.

under similar circumstances (Figure 2A), these adjusted delays are most similar to those of common marmosets (Stevens et al., 2005a).

Across primates, indifference point times vary by more than an order of magnitude from cotton-top tamarins to chimpanzees. What can account for this variation across species? One possibility is that animals simply maximize their rate of food intake. There are two ways in which individuals or species may have different indifference points when rate maximizing. First, they may vary in the time it takes to handle and consume food. Second, they may include different temporal components in the rate estimation (Mazur, 1994; Rosati et al., 2006; Stevens and Stephens, 2009; Pearson et al., 2010). Optimal foraging theory (Stephens and Krebs, 1986) assumes that animals maximize their long-term rate, so they should include all temporal components in their rate. In our study, that includes the delay, handling time, and intertrial interval. Animals may, however, ignore the intertrial interval and focus on temporal components that occur only between choice and consumption, resulting in short-term rate maximization (Stephens and Anderson, 2001). Though consistent 
Table 1: Individual subject data.

\begin{tabular}{|c|c|c|c|c|c|c|c|c|c|c|c|c|}
\hline Subject & Species* & Sex & Sessions & $\begin{array}{c}\text { Small } \\
\text { amount }\end{array}$ & $\begin{array}{c}\text { Large } \\
\text { amount }\end{array}$ & ITI & $\begin{array}{l}\text { Short } \\
\text { delay }\end{array}$ & $\begin{array}{l}\text { Long } \\
\text { delay }\end{array}$ & $\begin{array}{c}\text { Small } \\
\text { latency }\end{array}$ & $\begin{array}{c}\text { Large } \\
\text { latency }\end{array}$ & $\begin{array}{c}\text { Small } \\
\text { handling }\end{array}$ & $\begin{array}{l}\text { Large han- } \\
\text { dling }\end{array}$ \\
\hline Blümchen & RR & $\mathrm{F}$ & 22 & 2 & 6 & 30 & 0.0 & 16.6 & 0.7 & 1.6 & 3.3 & 17.9 \\
\hline Gustav & BW & M & 33 & 2 & 6 & 30 & 0.0 & 9.2 & 0.8 & 1.4 & 3.0 & 11.4 \\
\hline Ole & BW & M & 41 & 2 & 6 & 30 & 0.0 & 19.8 & 0.4 & 0.9 & 3.3 & 13.2 \\
\hline Püppi & BW & $\mathrm{F}$ & 56 & 2 & 6 & 30 & 0.0 & 24.6 & 1.9 & 1.6 & 4.2 & 16.4 \\
\hline Uta & BL & $\mathrm{F}$ & 22 & 2 & 6 & 30 & 0.0 & 14.8 & 0.1 & 0.5 & 2.0 & 18.8 \\
\hline Mean & & & 35 & 2 & 6 & 30 & 0.0 & 17.0 & 0.8 & 1.2 & 3.2 & 15.5 \\
\hline
\end{tabular}

*For species, R is red ruffed lemur, BW is black-and-white ruffed lemur, and BL is black lemur.

with intertemporal choices in other primates (Stevens et al., 2005a; Rosati et al., 2007), our analysis here indicates that neither short- nor long-term rate maximization models accounted for the intertemporal choice pattern observed in lemurs.

A number of other possible factors may influence intertemporal choice, ranging from metabolic rate and life expectancy to social structure and foraging ecology (reviewed in Stevens and Stephens, 2009; Addessi et al., 2011). Metabolic rate is relevant for intertemporal choice for food because the rewards directly feed into metabolism. Therefore, species with higher metabolisms need food more quickly and may opt for sooner rewards than those with lower metabolisms (Tobin and Logue, 1994). Life expectancy is also relevant because shorter life spans should make delayed rewards less beneficial, so shorter-lived species should choose sooner rewards (Stevens and Stephens, 2009). Unfortunately, we do not have good direct measures of metabolic rate and life expectancy for all of the primates analyzed here. Body size, however, offers a reasonable correlate for both metabolic rate and life expectancy: in mammals, larger species tend to have lower metabolisms and longer life expectancies (Speakman, 2005). Consequently, larger species may choose more delayed rewards than smaller species. In general, this is the case with the species shown in Figure 3 when examining the mean adjusted delay to indifference as a function of the log body mass-larger species wait longer than smaller species (Spearman correlation, $r_{s}=0.74$, $N=12, p=0.01)$. This result differs from Addessi et al.'s (2011) finding of no relationship, perhaps because this analysis both includes more species (using different experimental designs) and pools the two capuchin studies into a single data point. The two capuchin studies differ in their methodologies: Addessi et al. uses two versus six food items, whereas Amici et al. (2008) uses one versus three. Though the absolute magnitude of the rewards influences human intertemporal choices (e.g., 100 vs. 500 dollars is not the same as 1000 vs. 5000 dollars; Green et al., 1999), they have not been shown to in- fluence choices in animals (Kirby and Maraković, 1996; Green et al., 2004). Moreover, Stevens et al. (2005b) directly compared one versus three and two versus six food items in a spatial discounting task with tamarins and marmosets and found no effect of the reward magnitude. Thus, we pooled these data and observe that intertemporal choice correlates with body mass.

Lemurs fall below the regression line in this analysis, suggesting that they wait for shorter times than expected by their body size. Capuchins provide an interesting comparison because, though they share similar body sizes, capuchins wait on average three times longer (almost five times longer using only the data from Addessi et al., 2011) than lemurs. Though making comparisons across phylogenetically distantly related taxa warrants caution, socio-ecological characters such as social complexity, tool use, and extractive foraging for the lemur species tested here may not be as developed as those of capuchins (Jolly, 1998; Vasey, 2003). Without these pressures, lemurs may not have evolved the ability to wait like other primate species.

Though body size correlates with metabolic rate and life expectancy, it also correlates with many other factors such as diet and territory size. Therefore, rather than using body size as a proxy for other variables, we must measure those specific variables to assess their influence on temporal preferences. Moreover, in our data, body size also correlates with phylogenetic relationship. To control for phylogenetic relationship between species (Figure S1), we conducted phylogenetically independent contrasts with weighted branch lengths (Felsenstein, 1985) based on a primate phylogeny created from 10kTrees version 3 (Arnold et al., 2010) and the pigeon and rat branch lengths calculated using TimeTree (Hedges et al., 2006). Independent contrasts suggest that body size remains an influence on intertemporal choice even after accounting for phylogeny $\left(F_{1,10}=10.55, p=0.01\right.$, Figure S2).

Though large-scale relationships between intertemporal choice and body size and phylogeny exist across species, other factors such as social structure and for- 


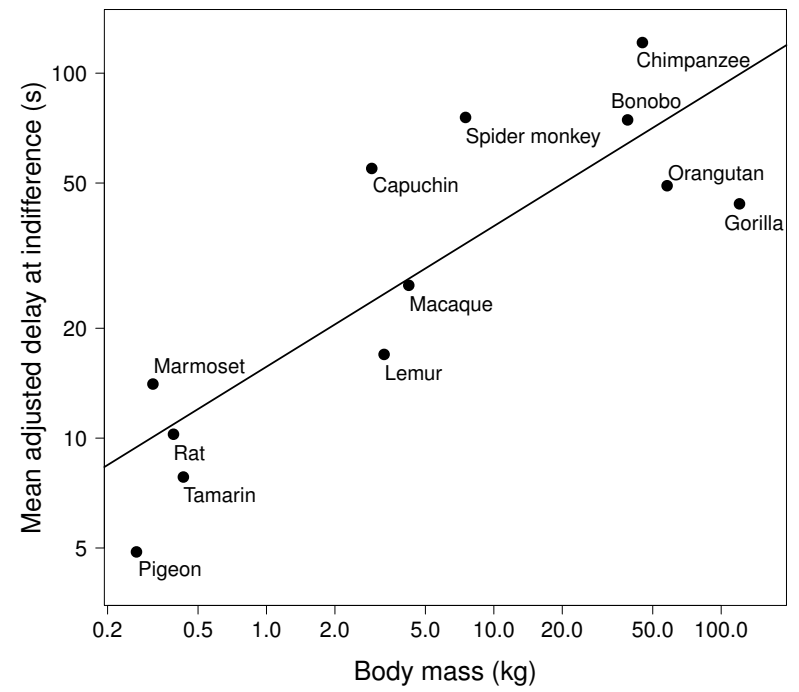

Figure 3: Body mass and intertemporal choice. We have pooled all capuchin and macaque subjects to generate a single mean adjusted delay for each species. Both $\mathrm{x}$ - and $\mathrm{y}$-axes are log scaled.

aging ecology may also influence intertemporal choice for more closely related species. Amici et al. (2008) proposed that social structure shapes choice via fissionfusion dynamics. In species with high fission-fusion dynamics, group members enter and leave the group frequently, resulting in a very fluid social structure. Amici et al. suggested that this turnover may select for more inhibitory control to suppress "prepotent but ineffective responses in a changing social environment" (p. 1415). That is, fission-fusion species require flexibility in inhibiting their social behavior and in turn may have more control over inhibitory processes in the food domain as well. When they tested the primate species in Figure 2B, Amici et al. reported that fission-fusion species tend to wait longer for food rewards, thereby confirming their predictions.

Foraging ecology offers another factor relevant to intertemporal choice. Species that frequently face delays in their natural foraging ecology (such as gummivores, stalking and sit-and-wait hunters, and extractive foragers) likely face selection pressure for the ability to wait in intertemporal choices (Stevens and Stephens, 2009). The ability to wait (or not) may be "ecologically rational" (Todd and Gigerenzer, 2007) depending on the temporal structure of the environment. Foraging ecology may act as an important factor explaining why common marmosets wait longer than tamarins and chimpanzees wait longer than bonobos (Stevens et al., 2005a; Rosati et al., 2007). Moreover, Addessi et al. (2011) proposed that the extractive foraging used by ca- puchins explains their long wait times in intertemporal choice tasks compared to other New World monkeys. Thus, the social and foraging environments likely exert strong selection pressure for intertemporal choices. Other, more internal, factors such as differential valuation of rewards and time perception may also influence choice. The factors mentioned here are not mutually exclusive, however, and likely combine to shape intertemporal choice.

\subsection{Conclusions}

In summary, lemurs' intertemporal choices match those of the callitrichid primates tested with similar methodologies. They do not appear to maximize their food intake rate in this task. A comparative analysis with 12 species suggests that intertemporal choices correlate with body size, even when accounting for phylogeny.

In this study, we tested too few species to make general claims about lemurs broadly because lemur species differ greatly in their social and ecological characters. For instance, in a comparative analysis of 19 species, MacLean et al. (2009) explored how pairbonding, group size, diet, and activity pattern relate to brain size in lemurs. Though the social factors did not relate to brain size in their analysis, the ecological factors did: frugivores had larger brains that foliovores and cathemeral species (those active both during day and night) had larger brains than diurnal species. The lemur species tested here are primarily frugivorous and diurnal. Comparisons with foliovorous and cathemeral species might result in interesting differences in waiting times. Despite the overall lack of an influence of group size on brain size in MacLean et al. (2009), studies of specific cognitive tasks do show differences in performance. For instance, the more highly social ring-tailed lemurs (Lemur catta) outperformed less social species such as mongoose lemurs (Eulemur mongoz), black lemurs, and ruffed lemurs in transitive inference and social cognition tasks (MacLean et al., 2008; Sandel et al., 2011). Lemur species vary widely in life history, ecology, and social structure, ranging from rather solitary, specialized extractive foragers such as aye-ayes (Daubentonia madagascariensis) to the fission-fusion and frugivorous ruffed lemurs (Morland, 1991; Vasey, 2006). This broad diversity among species makes lemurs a particularly interesting group for testing comparative hypotheses regarding factors influencing intertemporal choice. 


\section{Acknowledgments}

This article is in honor of the contributions of Alex Kacelnik to the study of animal decision making. We would like to thank the staff of Tierpark Berlin, Bernhard Blaszkiewitz, and Andreas Pauly for granting permission for and assistance with the project. We thank Federica Amici for allowing us to analyze her individual subject data, Elsa Addessi, Fabio Paglieri, and Alex Rosati for comments on the manuscript, and Evan MacLean and Charlie Nunn for advice on the comparative analysis. This work complies with the Animal Behavior Society's Guidelines for the Treatment of Animals in Behavioral Research and Teaching and the animal welfare laws of Germany.

\section{References}

Addessi, E., Paglieri, F., Focaroli, V., 2011. The ecological rationality of delay tolerance: insights from capuchin monkeys. Cognition $119,142-147$.

Amici, F., Aureli, F., Call, J., 2008. Fission-fusion dynamics, behavioral flexibility, and inhibitory control in primates. Current Biology $18,1415-1419$

Arnold, C., Matthews, L.J., Nunn, C.L., 2010. The 10kTrees website: a new online resource for primate phylogeny. Evolutionary Anthropology 19, 114-118.

Bateson, M., Kacelnik, A., 1996. Rate currencies and the foraging starling: the fallacy of the averages revisited. Behavioral Ecology 7, 341-352.

Beran, M.J., Savage-Rumbaugh, E., Pate, J.L., Rumbaugh, D.M., 1999. Delay of gratification in chimpanzees (Pan troglodytes). Developmental Psychobiology 34, 119-127.

Brunner, D., Hen, R., 1997. Insights into the neurobiology of impulsive behavior from serotonin receptor knockout mice. Annals of the New York Academy of Sciences 836, 81-105.

Charnov, E.L., 1976. Optimal foraging: the marginal value theorem. Theoretical Population Biology 9, 129-136.

Cheng, K., Penea, J., Porter, M.A., Irwin, J.D., 2002. Self-control in honeybees. Psychonomic Bulletin and Review 9, 259-263.

Dahl, D.B., 2009. xtable: export tables to LaTeX or HTML. R package version 1.5-6.

Eklund, A.C., 2010. beeswarm: the bee swarm plot, an alternative to stripchart. R package version 0.1.1.

Felsenstein, J., 1985. Phylogenies and the comparative method. American Naturalist 125, 1-15.

Genty, E., Palmier, C., Roeder, J., 2004. Learning to suppress responses to the larger of two rewards in two species of lemurs, $E u$ lemur fulvus and E. macaco. Animal Behaviour 67, 925-932.

Green, L., Myerson, J., 2004. A discounting framework for choice with delayed and probabilistic rewards. Psychological Bulletin 130, 769-792.

Green, L., Myerson, J., Holt, D.D., Slevin, J.R., Estle, S.J., 2004. Discounting of delayed food rewards in pigeons and rats: is there a magnitude effect? Journal of the Experimental Analysis of Behavior $81,39-50$.

Green, L., Myerson, J., Ostaszewski, P., 1999. Amount of reward has opposite effects on the discounting of delayed and probabilistic outcomes. Journal of Experimental Psychology: Learning, Memory, and Cognition 25, 418-427.
Green, L., Myerson, J., Shah, A.K., Estle, S.J., Holt, D.D., 2007. Do adjusting-amount and adjusting-delay procedures produce equivalent estimates of subjective value in pigeons? Journal of the Experimental Analysis of Behavior 87, 337-347.

Hedges, S.B., Dudley, J., Kumar, S., 2006. Timetree: a public knowledge-base of divergence times among organisms. Bioinformatics 22, $2971-2972$.

Herrnstein, R.J., 1961. Relative and absolute strength of response as a function of frequency of reinforcement. Journal of the Experimental Analysis of Behavior 4, 267-272.

Isler, K., Christopher Kirk, E., Miller, J.M., Albrecht, G.A., Gelvin, B.R., Martin, R.D., 2008. Endocranial volumes of primate species: scaling analyses using a comprehensive and reliable data set. Journal of Human Evolution 55, 967-978.

Jolly, A., 1998. Pair-bonding, female aggression and the evolution of lemur societies. Folia Primatologica 69, 1-13.

Kacelnik, A., 2003. The evolution of patience, in: Loewenstein, G., Read, D., Baumeister, R.F. (Eds.), Time and Decision: Economic and Psychological Perspectives on Intertemporal Choice. Russell Sage Foundation, New York, pp. 115-138.

Kangas, B.D., Branch, M.N., 2006. Stability of pigeon body weight under free-feeding conditions. Journal of the Experimental Analysis of Behavior 86, 393-396.

Kirby, K.N., Maraković, N.N., 1996. Delay-discounting probabilistic rewards: rates decrease as amounts increase. Psychonomic Bulletin and Review 3, 100-104.

de Leeuw, J., 2001. Reproducible research: the bottom line. Technical Report. Department of Statistics Papers, UCLA. Los Angeles.

Leisch, F., 2002. Sweave: dynamic generation of statistical reports using literate data analysis, in: Härdle, W., Rönz, B. (Eds.), Compstat 2002-Proceedings in Computational Statistics. Physica Verlag, Heidelberg, pp. 575-580.

Lewis, K.P., Jaffe, S., Brannon, E.M., 2005. Analog number representations in mongoose lemurs (Eulemur mongoz): evidence from a search task. Animal Cognition 8, 247-252.

MacLean, E.L., Barrickman, N.L., Johnson, E.M., Wall, C.E., 2009. Sociality, ecology, and relative brain size in lemurs. Journal of Human Evolution 56, 471-478.

MacLean, E.L., Mandalaywala, T.M., Brannon, E.M., 2011. Variance-sensitive choice in lemurs: constancy trumps quantity. Animal Cognition, in press.

MacLean, E.L., Merritt, D.J., Brannon, E.M., 2008. Social complexity predicts transitive reasoning in prosimian primates. Animal Behaviour 76, 479-486.

Mazur, J.E., 1987. An adjusting procedure for studying delayed reinforcement, in: Commons, M.L., Mazur, J.E., Nevin, J.A., Rachlin, H. (Eds.), Quantitative Analyses of Behavior: The Effect of Delay and of Intervening Events on Reinforcement Value. Lawrence Erlbaum Associates, Hillsdale, NJ. volume 5, pp. 55-73.

Mazur, J.E., 1994. Effects of intertrial reinforcers on self-control choice. Journal of the Experimental Analysis of Behavior 61, 8396.

McDiarmid, C.G., Rilling, M.E., 1965. Reinforcement delay and reinforcement rate as determinants of schedule preference. Psychonomic Science 2, 195-196.

Morland, H.S., 1991. Preliminary report on the social organization of ruffed lemurs (Varecia variegata variegata) in a northeast Madagascar rain forest. Folia Primatologica 56, 157-161.

Mühlhoff, N., Stevens, J.R., Reader, S.M., 2011. Spatial discounting of food and social rewards in guppies (Poecilia reticulata). Frontiers in Psychology 2, 68.

Paradis, E., Claude, J., Strimmer, K., 2004. APE: analyses of phylogenetics and evolution in R language. Bioinformatics 20, 289-290.

Pearson, J., Hayden, B., Platt, M., 2010. Explicit information reduces discounting behavior in monkeys. Frontiers in Psychology 1, 237. 
Perry, J.L., Larson, E.B., German, J.P., Madden, G.J., Carroll, M.E., 2004. Impulsivity (delay discounting) as a predictor of acquisition of IV cocaine self-administration in female rats. Psychopharmacology 178, 193-201.

R Development Core Team, 2011. R: A language and environment for statistical computing. http://www.R-project.org.

Ramseyer, A., Pele, M., Dufour, V., Chauvin, C., Thierry, B., 2006. Accepting loss: the temporal limits of reciprocity in brown capuchin monkeys. Proceedings of the Royal Society of London, Series B 273, 179-184.

Read, D., 2004. Intertemporal choice, in: Koehler, D., Harvey, N. (Eds.), Blackwell Handbook of Judgment and Decision Making. Blackwell, Oxford, pp. 424-443.

Richards, J.B., Mitchell, S.H., de Wit, H., Seiden, L.S., 1997. Determination of discount functions in rats with an adjusting-amount procedure. Journal of the Experimental Analysis of Behavior 67, 353-366.

Rosati, A.G., Stevens, J.R., Hare, B., Hauser, M.D., 2007. The evolutionary origins of human patience: temporal preferences in chimpanzees, bonobos, and adult humans. Current Biology 17, 16631668.

Rosati, A.G., Stevens, J.R., Hauser, M.D., 2006. The effect of handling time on temporal discounting in two New World primates. Animal Behaviour 71, 1379-1387.

Sandel, A.A., MacLean, E.L., Hare, B., 2011. Evidence from four lemur species that ringtailed lemur social cognition converges with that of haplorhine primates. Animal Behaviour 81, 925-931.

Santos, L.R., Barnes, J.L., Mahajan, N., 2005. Expectations about numerical events in four lemur species (Eulemur fulvus, Eulemur mongoz, Lemur catta and Varecia rubra). Animal Cognition 8, 253-262.

Skinner, B.F., 1938. The Behavior of Organisms: An Experimental Analysis. Prentice Hall, Englewood Cliffs, NJ.

Speakman, J.R., 2005. Body size, energy metabolism and lifespan Journal of Experimental Biology 208, 1717-1730.

Stephens, D.W., Anderson, D., 2001. The adaptive value of preference for immediacy: when shortsighted rules have farsighted consequences. Behavioral Ecology 12, 330-339.

Stephens, D.W., Krebs, J.R., 1986. Foraging Theory. Princeton University Press, Princeton.

Stevens, J.R., 2010. Intertemporal choice, in: Breed, M.D., Moore, J. (Eds.), Encyclopedia of Animal Behavior. Academic Press, Oxford. volume 2, pp. 203-208.

Stevens, J.R., Hallinan, E.V., Hauser, M.D., 2005a. The ecology and evolution of patience in two New World monkeys. Biology Letters $1,223-226$

Stevens, J.R., Rosati, A.G., Ross, K.R., Hauser, M.D., 2005b. Will travel for food: spatial discounting in two New World primates. Current Biology 15, 1855-1860.

Stevens, J.R., Stephens, D.W., 2009. The adaptive nature of impulsivity, in: Madden, G.J., Bickel, W.K. (Eds.), Impulsivity: The Behavioral and Neurological Science of Discounting. American Psychological Association, Washington, DC, pp. 361-387.

Tobin, H., Logue, A.W., 1994. Self-control across species (Columba livia, Homo sapiens, and Rattus norvegicus). Journal of Comparative Psychology 108, 126-133.

Tobin, H., Logue, A.W., Chelonis, J.J., Ackerman, K.T., 1996. Selfcontrol in the monkey Macaca fascicularis. Animal Learning and Behavior 24, 168-174.

Todd, P.M., Gigerenzer, G., 2007. Environments that make us smart: ecological rationality. Current Directions in Psychological Science $16,167-171$.

Vasey, N., 2003. Varecia, ruffed lemurs, in: Goodman, S.M., Benstead, J. (Eds.), The Natural History of Madagascar. University of Chicago Press, Chicago, pp. 1332-1336.
Vasey, N., 2006. Impact of seasonality and reproduction on social structure, ranging patterns, and fission-fusion social organization in red ruffed lemurs, in: Gould, L., Sauther, M.L. (Eds.), Lemurs: Ecology and Adaptation. Springer, Boston, MA, pp. 275-304.

Vick, S., Bovet, D., Anderson, J., 2010. How do African grey parrots (Psittacus erithacus) perform on a delay of gratification task? Animal Cognition 13, 351-358. 


\section{Supplementary Material}

Movie S1. Intertemporal choice test. Video recording of black lemur free-choice trials. In the first clip, the subject chose six apple pieces after $13 \mathrm{~s}$ and in the second clip she chose two apple pieces available immediately. Data sheet $S 1$. Lemur intertemporal choice data.

Data sheet $S 2$. Comparative intertemporal choice data. Individual subject intertemporal choice data for 12 animal species.

Data sheet $S 3$. R code for data analysis.

Figure S1. Phylogeny of species in comparative analysis.

Figure S2. Independent contrasts of body mass and intertemporal choice.

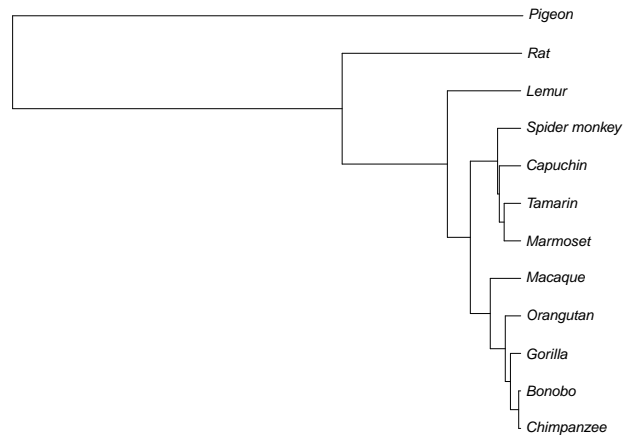

Figure S1: Phylogeny of species in comparative analysis. We used 10kTrees version 3 (http://10ktrees.fas.harvard.edu/index.html, Arnold et al., 2010) to construct the weighted branch lengths of the primate phylogeny and TreeTime (http://www.timetree.org/, Hedges et al., 2006) for the weighted branch lengths of pigeons and rats.

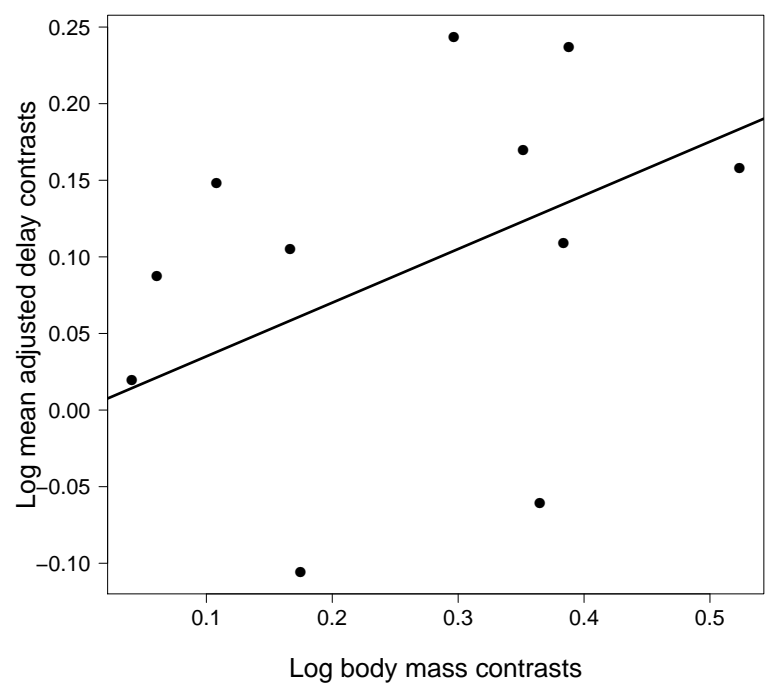

Figure S2: Independent contrasts of log body mass and intertemporal choice. When accounting for phylogeny, the relationship between intertemporal choice and $\log$ body size is maintained $(y=0.35 x$, $F_{1,10}=10.55, p=0.01$, adjusted $\left.R^{2}=0.46\right)$. 